\title{
The Clinical Applications of Endometrial Mesenchymal Stem Cells
}

\author{
Wanyun Zuo,, Bingyu Xie, Chenglong Li,, Yuhan Yan, Yangyi Zhang, \\ Wei Liu, ${ }^{2}$ Jufang Huang, ${ }^{3}$ and Dan $\mathrm{Chen}^{3}$
}

Endometrial mesenchymal stem cells (enMSCs) are a class of novel adult stem cells with self-renewal capacity, differentiation potential, low immunogenicity, low tumorigenicity, and other biological characteristics. Since the discovery of enMSCs, they have become a hot research topic. In recent years, research on enMSC isolation and application have made great progress. In this review, we focus on the clinical applications of this cell type. The latest research on the applications of enMSCs in the immune, gynecological, cardiovascular, digestive, nervous systems and metabolic diseases, as well as biobanking of enMSCs will be reviewed.

Keywords: endometrial mesenchymal stem cells, biological characteristics, animal model, clinical application, biobanking

\section{Introduction}

$\mathrm{M}$ ESENCHYMAL STEM CELls (MSCs) can be harvested from various human organs or tissues. With the rapid development of storage techniques, MSCs are becoming more convenient for research and clinical applications, although such applications are still restricted by limitations in collection techniques as well as ethical and legal restrictions. ${ }^{1-4}$

Chan reported the discovery of cells with strong multidirectional differentiation potential in the endometrium, the so-called endometrial MSCs (enMSCs). There are three kinds of stem cells in human endometrium: MSCs, epithelial stem cells, and endothelial stem cells. The CD146+PDGFR- $\beta+$ subpopulation comprise the enMSCs. ${ }^{5}$ Recently, researchers recommended W5C5 (SUSD2) as an alternative single marker to purify the same PDGF-R $\beta+/ C D 146+$ enMSC subpopulation. ${ }^{6}$ When setting a marker CD146+PDGFR- $\beta+C D 45-$ to identify the in vivo perivascular location for enMSCs, both the functionalis and basalis of human endometrium were positive ${ }^{7}$ indicating that enMSCs can also be derived from menstrual blood. ${ }^{8-10}$ Thus, these cells are also known as menstrual blood-derived stem cells (MenMSCs).

EnMSCs can be available potentially 12 times per year from reproductive age women, offering sufficient time to store cells for therapy. Compared with bone marrow mesenchymal stem cells (BMSCs), the noninvasive method for obtaining enMSCs is more acceptable for patients and can reduce their discomfort. ${ }^{11,12}$ Moreover, enMSCs show a greater multiplication ability. ${ }^{13,14}$ Among women 30-60 years of age, the younger the donors are, the stronger the multiplication ability. ${ }^{15}$ Studies have shown that enMSCs maintain a normal karyotype after 12 passages of subculture and retain their embryonic stem cell markers for up to 20 cycles of subculturing, or after infusion into a rat model for stroke. $^{12}$ In addition to the considerable multiplication ability, enMSCs have a strong potential in differentiation.

Evidence suggests that enMSCs exhibit a multilineage potential and have attracted extensive attention in regenerative medicine. Recent studies have shown enMSCs have a high rate of proliferation and possess multilineage differentiation capacity. Menstrual blood was introduced as an easily accessible stem cell source that can be isolated noninvasively from female volunteer donors with no ethical obstacles, making enMSCs an abundant and easily available source with no immunological response for cell replacement therapy. Many studies have shown that enMSCs can differentiate into many cell lines under standard culture conditions, including the epiblast, mesoblast, and hypoblast cells, such as fibroblast-like cells when treated with connective tissue growth factor, ${ }^{16}$ decidual cells, ${ }^{17}$ oocyte-like cells, pancreatic cells, ${ }^{7}$ Schwann cells, ${ }^{18}$ osteoblasts, ${ }^{19}$ endometrial epithelium cells, ${ }^{20}$ adipocytes, ${ }^{21}$ neurons, and hepatocyte-like cells. The potential for cell therapy is promising in nerve regeneration, inducing enMSCs entering into the neurons in vitro to provide an unlimited source of human neural cells and facilitate their clinical applications for neurological diseases. ${ }^{22}$ Studies have

\footnotetext{
${ }^{1}$ Xiangya School of Medicine, Central South University, Changsha, China.

${ }^{2}$ Institute of Human Reproductive and Stem Cell Engineering, Xiangya School of Medicine, Central South University, Changsha, China.

${ }^{3}$ Department of Anatomy and Neurobiology, Xiangya School of Medicine, Central South University, Changsha, China.
} 
also compared the hepatic differentiation ability of enMSCs with BMSCs, taking accessibility, refreshing nature, and the lack of ethical issues into account, and concluded that enMSCs could be considered a safe alternative to BMSCs for future stem cell therapy for chronic liver diseases. ${ }^{23}$ Moreover, enMSCs can differentiate to epidermal cells that can be used noninvasively in various dermatological lesions and diseases. $^{24}$ Since enMSCs can transform into oocyte-like cells, they may potentially represent an in vitro system for the investigation of human folliculogenesis. ${ }^{25}$ Based on the overall results from prior research, we suggest that enMSCs could represent a new promising tool having potential within cell therapy applications. Thus, the clinical application of enMSCs is the focus of this review.

Studies have shown that MSCs derived from the umbilical cord matrix, adipose tissue, and bone marrow have different abilities to inhibit $\mathrm{B}$ and $\mathrm{T}$ lymphocytes and natural killer cells in peripheral blood. ${ }^{26}$ MSCs can also have immunosuppressive effects on other immunocompetent cells such as dendritic cells (DC) by affecting the DC recruitment, maturation, and function. ${ }^{27}$ These immunomodulatory capabilities are generated by releasing inflammatory cytokines into the tissue microenvironment. ${ }^{28}$ Secreting immunosuppressive cytokines such as interferon-gamma (IFN- $\gamma$ ) and tumor necrosis factor-alpha make MSCs detrimental for both innate immunity and adaptive immunity. ${ }^{29-32}$ However, as a newly discovered category of MSCs, the capabilities of enMSCs in immunology are not very clear. A few studies show that enMSCs have the characteristics of low immunogenicity and immunosuppressive effects, ${ }^{33-35}$ the key to clinical applications.

\section{Research on Clinical Applications}

\section{Application of enMSCs in the immune system}

The investigations from Zhou determined that enMSCs had a low expression of HLA-ABC while HLD-DR was negative, indicating that enMSCs have low immunogenicity. When cocultured with mouse spleen lymphocytes or human peripheral blood lymphocytes, enMSCs can inhibit their proliferation and significantly suppress the IFN- $\gamma$ secretion of human peripheral blood lymphocytes, which may be induced by TGF- $\beta 1$ secreted by enMSCs. ${ }^{33}$ Subsequent research found that when cocultured with peripheral blood mononuclear cells (PBMCs), enMSCs play a dual role, which depends on the ratio between enMSCs and PBMCs. At a higher ratio $(1: 1$ to $1: 2)$, enMSCs inhibited the proliferation capacity of PBMCs, while at lower ratios $(1: 32$ to $1: 64)$ they promoted proliferation. Further studies are required to determine the molecules and mechanisms responsible for this dose-dependent effect. ${ }^{34}$ Mahmood et al. cocultivated enMSCs and monocytes in a report from 2014. There is a significantly decreased level of CD1a+ (iDC specific) cells and an increased level of CD14+ (monocyte specific) cells in the cocultivated group than in the mDConly (enMSC-DC) group, showing that the stem cells could inhibit the differentiation of monocytes toward iDCs. ${ }^{35}$ They also measured the concentration of cytokines, finding that the levels of IL-6 and IL-10 had a noticeable increase in the coculture groups, suggesting enMSCs could inhibit the generation and maturation of iDCs via secreting immunosuppressive cytokines.
Experiments have been conducted to confirm that enMSCs have low immunogenicity, ${ }^{33}$ which is an important foundation to allow enMSCs to be used in clinical applications. With their low immunogenicity, when enMSCs are injected into the human body, they have weaker immunological effects and prevent immune injury. These results have encouraged us to suggest the use enMSCs as a new stem cell source in stem cell therapy for premature ovarian failure (POF) ${ }^{36}$ diabetes, ${ }^{37}$ Parkinson's disease, ${ }^{38}$ liver cirrhosis, ${ }^{39}$ myocardial infarction ${ }^{40}$ and so on. Another important immunological characteristic of enMSCs is the immunosuppressive effects similar to other MSCs, ${ }^{35}$ and combined with their low immunogenicity advantage, enMSCs in the future may be used to suppress autoimmunity or harmful excessive immune responses, and thus applied in treatment of autoimmune diseases, inflammation, and organ or bone marrow transplantation.

\section{Application of enMSCs in the gynecological system}

With its multidirectional differentiation potential and the advantage of similar tissue specificity, enMSCs are considered a good source for conducting stem cell therapy for gynecological diseases, especially certain tissue damage that is difficult to repair.

It has been demonstrated that enMSCs are capable of differentiation into endometrial epithelium and stromal cells in vitro under the induction of estradiol. ${ }^{41}$ When implanted subcutaneously in nonobese diabetic/severe combined immune deficiency female mice, enMSCs were found to reconstruct endometrial tissue. ${ }^{42}$ Both endometrium thickness and microvessel density (MVD) increased after implantation of enMSCs in a mouse endometrial injury model. ${ }^{43}$ When enMSCs were injected into a POF mouse model, they could survive and express specific proteins of ovarian granulosa cells, indicating that enMSCs can differentiate into granulosa-like cells. ${ }^{4}$ Furthermore, ovarian weight, estradiol levels, and the number of normal follicles were higher than in the control group. Dongmei also found that the estrous cycle and body weight of the mice were increased and green fluorescence was detected in the ovarian stroma after injecting green fluorescent protein-labeled enMSCs. ${ }^{45}$ Meanwhile, the increasing estradiol levels can also promote the development of follicles. The five experiments above demonstrate that enMSCs have the potential to differentiate to endometrial tissue and granulosa-like cells in vitro, and their effects on reconstruction and repair in vivo as shown in mouse models. Patients with intrauterine adhesion were found to have fewer enMSCs than healthy controls by immunohistochemistry under hysteroscope, also revealing that enMSCs play an important role in maintaining the structure and function of the endometrium. ${ }^{41}$ In 2017 , Wang et al. proved that enMSCs could repair premature ovary in mice, suggesting that transplantation of enMSCs may provide an effective and novel strategy for treating POF. ${ }^{36}$ In addition, Zhang discovered that enMSCs have the ability to identify sites of injury and direct migration. ${ }^{43}$ This feature suggests that it is advantageous to implant enMSCs through the veins instead of through the endometrium, and thus reduce damage to the patient in clinical applications. The studies on tissue injury have progressed only in animal models. To date, studies have only shown a phenomenon, but nothing related to the mechanism or the potential risks 
such as tumorigenicity or risk of causing endometriosis. Many details remain to be assessed before clinic trials become appropriate.

Under the background concerning intrinsic antitumor properties of MSCs against various kinds of cancer, ${ }^{46-49} \mathrm{Bu}$ et al. conducted in vitro and/or in vivo experiments to determine whether enMSCs have innate antitumor properties on epithelial ovarian cancer (EOC) cells. ${ }^{50}$ The results show that enMSCs can attenuate tumor growth in various ways in in vitro and/or in vivo experiments. This supports the hypothesis and provides a new strategy for therapy of EOC. With its properties against EOC, enMSCs may be used for various tumors.

In 2003, Wu et al. showed epithelial cells of endometrium ectopic foci to be monoclonal, suggesting that stem cells may play a role. ${ }^{51}$ Chan et al. showed that ectopic endometrial lesions contain cells with the feature of enMSCs, in 2011. ${ }^{52}$ All of the evidence supports the view that the enMSCs play an important part in the formation of endometrial ectopic lesions. In the formation of ectopic endometrial lesions, blood vessel formation is the key. Yerlikaya et al. found that patients' angiogenesis factors such as vascular endothelial growth factor receptor (VEGFR) and platelet-derived growth factor subunit $\mathrm{B}$ in endometrial ectopic foci express more highly than in eutopic endometrium, and higher in eutopic endometrium of patients than healthy subjects. ${ }^{53}$ From this research, another way enMSCs can repair tissues was found to be through the promotion of angiogenesis. Their secretion products may be helpful in the repair of damaged tissues. The specific mechanisms of endometriosis and the role of enMSCs remain to be further studied. Angiogenesis and cell migration are believed to be the most important processes to form an endometrial ectopic lesion. Based on the above research, we can propose a hypothesis that enMSCs migrate to the ectopic foci and secrete factors to promote angiogenesis, and thus form endometrial tissue. In addition, Proestling et al. found that the epithelial expression of OCT4, SOX15, and TWIST1 is increased in the ectopic lesions compared to the eutopic endometrium and patients without endometriosis, revealing that upregulation of these markers contributes to the establishment of ectopic endometriotic lesions. ${ }^{54}$ Upregulation of these stemness-related factors also supports the hypothesis that enMSCs are the key to the treatment of endometriosis.

\section{Application of enMSCs in the cardiovascular system}

In 2008, Hida et al. transplanted enMSCs into the infarcted region of nude mice, and found that enMSCs differentiated into cardiomyocytes. Compared with the control group, the infarct size of the transplanted enMSC group decreased significantly. The results proved enMSCs have a significant effect on improving recovery from myocardial infarction. ${ }^{40}$ Subsequently, Jiang et al. injected enMSCs into the infarcted zone of the rat myocardial ischemic model. Compared with the control group, the cardiac function of the transplanted group was significantly improved, the density of the heart blood vessel was higher, and the transplantation of enMSCs promoted the regeneration of the cardiomyocytes. Meanwhile, enMSCs and cardiomyocytes were cocultured in a transwell plate. As a result, enMSCs mainly promoted the proliferation of existing cardiomyocytes through paracrine cytokines. ${ }^{55}$ Later, Jiang et al. directly extracted and enriched an enMSC-derived cytokine cocktail (EdCC) from enMSC culture medium to improve myocardial ischemia/reperfusion injury. The results showed that EdCC could significantly inhibit the expression of cleaved caspase 3 in the myocardial cells, demonstrating the antiapoptotic effect of EdCC from both cellular and protein levels. ${ }^{56}$ The paracrine products of enMSCs are safer and more efficient than enMSCs, and therefore more suitable for clinical use. This suggests that we should pay more attention to the efficacy of the secretions of enMSCs. Jiang et al. also studied the function and mechanism of MSCs from three different sources (bone marrow, adipose, and endometrium) as therapeutic agents for the treatment of cardiovascular diseases. ${ }^{55}$ They found that enMSCs provided better cardiac protection related to BMSCs or adipose MSCs and supported enhanced MVD. ${ }^{57}$ However, their study did not use enMSCs for clinical applications. However, in 2010, Ichim et al. injected enMSCs into a congestive heart failure patient's body by intravenous injection and found the patient's ejection fraction increased from $30 \%$ to $40 \%$, with Pro-BNP reduced. ${ }^{58}$ This is the only reference found in the literature concerning enMSCs for clinical use in cardiovascular disease. There are two possible reasons for this lack of information. First, the current research on enMSCs is not very thorough. Second, there is too much uncertainty concerning enMSCs for clinical use, such as how to control the tumorigenicity of enMSCs. For these reasons, researchers have paid more attention to the secretion of enMSCs.

\section{Application of enMSCs in the digestive system}

EnMSCs are changed to hepatic-like morphology and form a 3D structure when being cultured in hepatic differentiation media or being cocultured with liver homogenate in vitro, imitating hepatic-like function. The expression of albumin (ALB) and $\alpha$-fetoprotein (AFP) is higher when being cocultured with homogenate from damaged liver tissue, indicating the clinical potential to repair liver injury. ${ }^{59}$ In vitro animal experiments were conducted in Zhejiang University to explore the mechanism in the treatment of liver cirrhosis in 2015. EnMSCs were monitored migrating to the damaged region in the induced hepatic fibrosis mice, whereas almost no hepatic-like cells that differentiated from enMSCs were found. However, LX-2, an immortalized hepatic stellate cell line that is the main source of fibroblasts, was obviously reduced when cocultured with enMSCs. ${ }^{39}$ Thus, enMSC function was verified to reduce the amount of collagen deposition, and the proliferation ability of LX-2 was weakened due to enMSC paracrine effect. However, this finding is too early to be applied in the clinic, because there is not sufficient evidence from animal studies about the inhibitory effect on LX-2 in vivo.

Previous treatments for ulcerative colitis (UC) are based on anti-inflammatory therapy, inducing immune tolerance, which leads to the result that patients miss the optimal period for surgery, along with side effects such as the development of metabolic syndromes. ${ }^{60} \mathrm{UC}$ might be cured with enMSC tissue repair healing capacity, depending on the potential of self-renewal and multidirectional differentiation. ${ }^{61}$ Although there have been no in vitro experiments to improve the differentiation of enMSCs to intestinal mucosal epithelial cells, enMSCs can interact with immune cells with soluble factor secretion. ${ }^{62}$ From animal testing, the researchers induced UC mice by injecting dextran sulfate sodium. The enMSC-treated group was shown to produce an obvious curative effect for 
UC in comparison with the untreated group. Despite that, the treated group failed to reach the state of the healthy group completely. ${ }^{63}$ However, the results suggested that enMSCs may also be effective for other inflammatory mediatorinduced diseases. Since the repair mechanism has not been explored completely, the consequences of clinical application of UC cannot be predicted.

\section{Application of enMSCs in the nervous system}

Stroke is a serious nervous system disease and it is a threat to human life and the quality of life. Tissue plasminogen activator is currently the best available therapeutic agent for stroke in clinical settings. However, the therapeutic window for the administration of the drug is limited to 3 hours after the onset of symptoms, ${ }^{64,65}$ and so, the patient usually cannot receive timely treatment. Borlongan et al. developed a stroke model through oxygen glucose deprivation (OGD) and cocultured enMSCs with OGDexposed primary rat neurons in vitro. They found enMSCs can reduce the death of these neurons. In further experiments, they transplanted enMSCs into rats with stroke, which also demonstrated the neuroprotective and neurogenesis effects of enMSCs. ${ }^{66}$ In 2012, Rodrigues and Borlongan et al. found that enMSCs promoted neuroprotection especially through modulation of the activated immune system and secretion of neurotrophic factors. ${ }^{67}$ In addition to the treatment of stroke, enMSCs were used for treatment of Parkinson's disease. Wolff et al. induced differentiation of enMSCs into dopaminergic neuron-like cells and transplanted them into a Parkinson's rat model. They found that these cells migrated to the lesion site of the rat and produced dopamine, demonstrating that enMSCs could effectively repair damaged neurons through cell replacement and by promoting endogenous repair. ${ }^{38}$ In 2015 , Wolff used St. Kitts green monkeys as test subjects, and found that enMSCs differentiated into a small number of tyrosine hydroxylasepositive cells and improved striatal dopamine production. ${ }^{68}$ Although cell and animal experiments have demonstrated that enMSCs can repair damaged neurons to treat stroke and Parkinson's disease, there are only a few clinical reports. In 2009, one study mentioned the effect of enMSCs in the treatment of gliomas. The tumor size in mice was reduced by $50 \%$ after injecting enMSCs in a mouse glioma model, probably because enMSCs have an antiangiogenic effect. ${ }^{69}$ However, no further study of treatment of gliomas with enMSCs was found. ${ }^{46}$ Also, in a previous article, we have repeatedly mentioned enMSC function to promote angiogenesis. ${ }^{51}$ The argument in this article contradicts the preceding text. ${ }^{53}$ Perhaps in different circumstances the treatment mechanism is different. At present, there is almost no clinical research concerning enMSCs in the treatment of neurological diseases in China or elsewhere. The most important reason is the effect of the blood-brain barrier on enMSC treatment of nervous system diseases. However, the current experiments in cells and animal models have shown a good prospect for using enMSCs for the nervous system disease.

\section{Application of enMSCs in metabolic disease}

Meng et al. induced enMSCs in vitro to differentiate into three germ lineages, including pancreatic in 2007. ${ }^{9}$ The emergence of enMSCs helped relieve the dilemma of stem cell acquisition problems. EnMSCs were applied to treat STZ (streptozotocin)-induced type I diabetes mice in 2014. All studied indexes, including weight and blood sugar, proved the effect of enMSCs on the treatment of diabetes. Further studies have confirmed that enMSCs can reverse the hyperglycemic state and restore islet structure in vivo. It is worth noting that the mechanism is not to replace the damaged B cells by enMSCs, but to promote the differentiation of progenitor cells, which is presumably accomplished through the secretion of cytokines. An in-depth study of this mechanism has great clinical value. ${ }^{37}$ The pathogenesis of diabetes is not simple, so putting stem cell therapy into clinical practice is premature. Moreover, we need to know more about stem cell therapy, such as the functional stability or the side effects on other organs. There will be a long time interval before enMSCs can be used in clinical applications.

\section{Biobanking of enMSCs}

Biobanks aim to perform research on biological samples collected from a variety of tissues and biological sample types. With the continuous development of cell therapy, such therapy has become an indispensable part of regenerative medicine. ${ }^{70}$ However, there are still some problems with cell therapy, such as cell engraftment and survival, stem cell fate control, and donor/patient compatibility for allogenic applications. ${ }^{71}$ Cell therapy still has significant application prospects. Using stem cells as the source materials for cell therapy, more and more research has begun to focus on stem cell collection. Therefore, stem cell banks have received much attention as a new biologic resource for both research and clinical applications. Stem cell banks represent a collection of biological materials (e.g., embryos and somatic tissues) and the associated data stored within an organized system. MSCs are spindle-shaped, plastic-adherent cells isolated from bone marrow, adipose tissue, dental pulp, and many other tissue sources. ${ }^{72,73}$ They play a therapeutic role mainly through differentiation and paracrine mechanisms. They are primarily mediated by a paracrine mechanism independent of cell differentiation. ${ }^{74}$ As a kind of MSC, enMSCs also provide therapeutic benefits through paracrine mechanisms independent of cell differentiation. This means that enMSCs have great potential for clinical use. The purpose of this article is not only to acknowledge the clinical application of enMSCs but more importantly to encourage the establishment of enMSC biobanks as well. As described above, enMSCs have the advantages of rich sources, abundancy, noninvasive collection methods, significant proliferation and differentiation potential, low immunogenicity, and no ethical controversy. Therefore, it is very useful to build a biobank of enMSCs.

However, establishing enMSC biobanks will be an arduous job. To establish a biobank for enMSCs, we should know how to separate, extract, identify, and cryopreserve enMSCs. Cell sources and isolation processes vary greatly. EnMSCs are derived from two sources: endometrium and menstrual blood. ${ }^{8-10}$ Extraction and preservation technology has matured significantly, which is the basis for the establishment of enMSC biobanks. One approach is to encourage menstruating women to store their own enMSCs in biobanks, to be used for the future treatment of their own tissue damage or as a new cell source for stem cell therapy or for 
scientific research. Another approach is to collect the endometrium. MSCs have some common characteristics that include fibroblast-like shape in culture, multipotent differentiation, extensive proliferation capacity, plastic adherence, and a common surface marker profile (e.g., CD34-, CD45-, CD31-, CD44+, CD90+, CD166+, and CD105+). ${ }^{70}$ Therefore, we can use these markers to identify and separate enMSCs. As for cryopreservation, enMSCs are the same as other MSCs. Slow freezing protocols utilizing dimethyl sulfoxide (DMSO) as the cryoprotectant are in use by many groups. ${ }^{75}$ Many studies have determined the optimal cryoprotective agent and concentration for preserving cells, and standard protocols include a 5\%-10\% DMSO solution. ${ }^{75-77}$ The second step to establish enMSC biobanks is to solve the problem of cell source. The collection of umbilical cord blood has become very common, and many umbilical cord blood stem cell banks have been established. However, enMSCs are a kind of novel stem cells whose advantages are not known from earlier research. It is important to strengthen the promotion of using enMSCs, which attracts people to collect and donate menstrual blood or endometrium. As for the donors, they should be healthy women of 25-35 years of age. To obtain high-quality cells, the donors need to have a health assessment before menstrual blood collection. The examination would include checking for infectious diseases such as AIDS and syphilis and gynecological diseases such as endometriosis, cervicitis, and pelvic inflammatory disease. The former conditions could lead to infectious diseases, while the latter disease conditions could affect the quality of the cells. The most important principles for establishing enMSC biobanks are to protect the autonomy and privacy of donors and ensuring safety through traceability. ${ }^{78,79}$

\section{Outlook}

The potential of multidirectional differentiation of enMSCs suggests its potential for repair of various types of tissue injury. Many reports in the literature have shown that stem cells can repair the myocardial tissue, endometrial tissue, ovarian tissue, and liver tissue in animal models. There is only preliminary information on the repair mechanism of enMSCs. Compared with other MSCs, enMSCs have low immunogenicity and tumorigenicity and the expectation of reducing the prevalence of diseases after further research.

At present, animal experiments and a few clinical cases in China and elsewhere reveal that the clinical application of enMSCs has a promising future. There is no doubt that enMSC biobanks will be of great significance for the treatment of various diseases and the development of stem cell therapy.

\section{Acknowledgments}

This work is supported by the following grants: grant sponsor: the National Key Research and Development Program of China, grant no. 2016YFC1201800; grant sponsor: the National Natural Science Foundation of China, grant no. 81400399; grant sponsor: the Project of Innovation-driven Plan of Central South University, grant no. 2015CXS022.

\section{Author Disclosure Statement}

No conflicting financial interests exist.

\section{References}

1. Yong KW, Wan SW, Xu F, et al. Cryopreservation of human MSCs for clinical applications: Current methods and challenges. Biopreserv Biobank 2015;13:231-239.

2. Campagnoli C, Roberts IA, Kumar S, et al. Identification of mesenchymal stem/progenitor cells in human first-trimester fetal blood, liver, and bone marrow. Blood 2001;98:23962402.

3. Zuk PA, Zhu M, Mizuno H, et al. Multilineage cells from human adipose tissue: Implications for cell-based therapies. Tissue Eng 2001;7:211-228.

4. Young HE, Steele TA, Bray RA, et al. Human reserve pluripotent MSCs are present in the connective tissues of skeletal muscle and dermis derived from fetal, adult, and geriatric donors. Anat Rec 2001;264:51-62.

5. Xu Y, Zhu H, Zhao D, Tan J. Endometrial stem cells: Clinical application and pathological roles. Int J Clin Exp Med 2015;8:22039-22044. eCollection 2015. Review.

6. Mutlu L, Hufnagel D, Taylor HS. The endometrium as a source of MSCs for regenerative medicine. Biol Reprod 2015;92:138.

7. Gargett CE, Schwab KE, Deane JA. Endometrial stem/ progenitor cells: The first 10 years. Hum Reprod Update 2016;22:137-163.

8. Chan RW, Schwab KE, Gargett CE. Clonogenicity of human endometrial epithelial and stromal cells. Biol Reprod 2004;70:1738-1750.

9. Meng X, Ichim TE, Zhong J, et al. Endometrial regenerative cells: A novel stem cell population. J Transl Med 2007; 15:57.

10. Du X, Yuan Q, Qu Y, et al. Endometrial MSCs isolated from menstrual blood by adherence. Stem Cells Int 2016: 3573846

11. Ghobadi F, Mehrabani D, Mehrabani G. Regenerative potential of endometrial stem cells: A mini review. World J Plast Surg 2015;4:3-8.

12. Allickson JG, Sanchez A, Yefimenko N, et al. Recent studies assessing the proliferative capability of a novel adult stem cell identified in menstrual blood. Open Stem Cell J 2011;3:4-10.

13. Troyer DL, Weiss ML. Wharton's jelly-derived cells are a primitive stromal cell population. Stem Cells 2008;26: 591-599.

14. Wang M, Yang Y, Yang D, et al. The immunomodulatory activity of human umbilical cord blood-derived MSCs in vitro. Immunology 2009;126:220-232.

15. Mehrabani D, Nazarabadi RB, Kasraeian M, et al. Growth kinetics, characterization, and plasticity of human menstrual blood stem cells. Iran J Med Sci 2016;41: $132-139$.

16. Su K, Edwards SL, Tan KS, et al. Induction of endometrial MSCs into tissue-forming cells suitable for fascial repair. Acta Biomater 2014;10:5012-5020.

17. Murakami K, Lee YH, Lucas ES, et al. Decidualization induces a secretome switch in perivascular niche cells of the human endometrium. Endocrinology 2014;155:45424553.

18. Bayat N, Ebrahimibarough S, Ardakan MM, et al. Differentiation of human endometrial stem cells into schwann cells in fibrin hydrogel as 3D culture. Mol Neurobiol 2016; 53:7170-7176.

19. Rossignoli F, Caselli A, Grisendi G, et al. Isolation, characterization, and transduction of endometrial decidual tissue multipotent mesenchymal stromal/stem cells from menstrual blood. Biomed Res Int 2013;2013:901821. 
20. Chen B, Obstetrics DO, Hospital HM. Endometrial stromal stem cells from early-pregnancy decidua differentiate into endometrial epithelial cells. Chinese J Tissue Eng Res 2016; 14:2098-2103.

21. Ai J, Shahverdi AR. Derivation of adipocytes from human Endometrial Stem Cells (EnSCs). J Reprod Infertil 2012; 13:151.

22. Noureddini M, Verdi J, Mortazavitabatabaei SA, et al. Human endometrial stem cell neurogenesis in response to NGF and bFGF. Cell Biol Int 2012;36:961-966.

23. Khanjani S, Khanmohammadi M, Zarnani AH, et al. Comparative evaluation of differentiation potential of menstrual blood- versus bone marrow- derived stem cells into hepatocyte-like cells. PLoS One 2014;9:e86075.

24. Faramarzi H, Mehrabani D, Fard M, et al. The potential of menstrual blood-derived stem cells in differentiation to epidermal lineage: A preliminary report. World J Plast Surg 2016;5:26-31.

25. Lai D, Guo Y, Zhang Q, Chen Y, Xiang C. Differentiation of human menstrual blood-derived endometrial MSCs into oocyte-like cells. Acta Biochim Biophys Sin 2016;48:9981005.

26. Ribeiro A, Laranjeira $\mathrm{P}$, Mendes S, et al. MSCs from umbilical cord matrix, adipose tissue and bone marrow exhibit different capability to suppress peripheral blood B, natural killer and T cells. Stem Cell Res Ther 2013;4:125.

27. Spaggiari GM, Abdelrazik H, Becchetti F, et al. MSCs inhibit monocyte-derived DC maturation and function by selectively interfering with the generation of immature DCs: Central role of MSC-derived prostaglandin E2. Blood 2009; 113:6576-6583.

28. Bernardo ME, Fibbe WE. Mesenchymal stromal cells: Sensors and switchers of inflammation. Cell Stem Cell 2013;13:392-402.

29. Krampera M, Cosmi L, Angeli R, et al. Role for interferongamma in the immunomodulatory activity of human bone marrow MSCs. Stem Cells 2006;24:386-398.

30. Corcione A, Benvenuto F, Ferretti E, et al. Human MSCs modulate B-cell functions. Blood 2006;107:367-372.

31. Spaggiari GM, Capobianco A, Becchetti S, et al. Mesenchymal stem cell-natural killer cell interactions: Evidence that activated NK cells are capable of killing MSCs, whereas MSCs can inhibit IL-2-induced NK-cell proliferation. Blood 2006;107:1484-1490.

32. Yu P, Xiao L, Lin L, et al. STAT3-mediated TLR2/4 pathway upregulation in an IFN-gamma-induced Chlamydia trachomatis persistent infection model. Pathog Dis 2016;74:ftw076.

33. Zhou C, Yang B, Tian Y, et al. Immunomodulatory effect of human umbilical cord Wharton's jelly-derived mesenchymal stem cells on lymphocytes. Cell Immunol 2011;272:33-38.

34. Nikoo S, Ebtekar M, Jeddi-Tehrani M, et al. Effect of menstrual blood-derived stromal stem cells on proliferative capacity of peripheral blood mononuclear cells in allogeneic mixed lymphocyte reaction. J Obst Gynaecol Res 2012; 38:804-809.

35. Mahmood B, Seyed Mohammad M, Mojdeh S, et al. Menstrual blood-derived stromal stem cells inhibit optimal generation and maturation of human monocyte-derived dendritic cells. Immunol Lett 2014;162(2 Pt B):239-246.

36. Wang Z, Wang Y, Yang $\mathrm{T}$, et al. Study of the reparative effects of menstrual-derived stem cells on premature ovarian failure in mice. Stem Cell Res Ther 2017;8:11.

37. Wu X, Luo Y, Chen J, et al. Transplantation of human menstrual blood progenitor cells improves hyperglycemia by promoting endogenous progenitor differentiation in type 1 diabetic mice. Stem Cells Dev 2014;23:1245-1257.

38. Sheng Xia Z. Cases of Endometrial Stem Cell Transplantation in the Treatment of Severe Intrauterine Adhesions in Preclinical Studies [D]. Ph.D. diss., Anhui Medical University, China; 2015. [in Chinese].

39. Wolff EF, Gao XB, Yao KV, et al. Endometrial stem cell transplantation restores dopamine production in a Parkinson's disease model. J Cell Mol Med 2011;15:747-755.

40. Masuda H, Matsuzaki Y, Hiratsu E, et al. Stem celllike properties of the endometrial side population: Implication in endometrial regeneration. PLoS One 2010;5: e10387.

41. Chen L, Zhang C, Chen L, et al. Human menstrual bloodderived stem cells ameliorate liver fibrosis in mice by targeting hepatic stellate cells via paracrine mediators. Stem Cells Transl Med 2017;6:272-284.

42. Zhang Y, Lin X, Dai Y, et al. Endometrial stem cells repair injured endometrium and induce angiogenesis via AKT and ERK pathways. Reproduction 2016;152:389-402.

43. Hida N, Nishiyama N, Miyoshi S, et al. Novel cardiac precursor-like cells fromHuman menstrua; blood derived mesenchymal cells. Stem Cells 2008;26:1695-1704.

44. Te Liu, Yongyi Huang, Jian Zhang, et al. Transplantation of human menstrual blood stem cells to treat premature ovarian failure in mouse model. Stem Cells Dev 2014;23: $1548-1557$.

45. Lai D, Wang F, Yao X, et al. Human endometrial MSCs restore ovarian function through improving the renewal of germline stem cells in a mouse model of premature ovarian failure. J Transl Med 2015;13:155.

46. Tang YM, Bao WM, Yang JH, et al. Umbilical cordderived MSCs inhibit growth and promote apoptosis of HepG2 cells. Mol Med Rep 2016;14:2717-2724.

47. Lin HD, Fong CY, Biswas A, et al. Human Wharton's jelly stem cells, its conditioned medium and cell-free lysate inhibit the growth of human lymphoma cells. Stem Cell Rev 2014;10:573-586.

48. Gauthaman K, Yee FC, Cheyyatraivendran S, et al. Human umbilical cord Wharton's jelly stem cell (hWJSC) extracts inhibit cancer cell growth in vitro. J Cell Biochem 2012; 113:2027-2039

49. Zhu P, Chen M, Wang L, et al. Systemic MSCs reduce growth rate of cisplatin-resistant ovarian cancer. Int J Clin Exp Pathol 2013;6:2506-2514.

50. Bu S, Wang Q, Zhang Q, et al. Human endometrial MSCs exhibit intrinsic anti-tumor properties on human epithelial ovarian cancer cells. Sci Rep 2016;6:37019.

51. Wu Y, Basir Z, Kajdacsy-Balla A, et al. Resolution of clonal origins for endometriotic lesions using laser capture microdissection and the human androgen receptor(HUMARA) assay. Fertil Steril 2003;79(Suppl 1):710 717.

52. Chan RW, Ng EH, Yeung WS. Identification of cells with colony-forming activity, self-renewal capacity, and multipotency in ovarian endometriosis. Am J Pathol 2011;178: 2832-2844.

53. Yerlikaya G, Balendran S, Pröstling K, et al. Comprehensive study of angiogenic factors in women with endometriosis compared to women without endometriosis. Eur J Obstet Gynecol Reprod Biol 2016;204:88-98.

54. Proestling K, Birner P, Balendran S, et al. Enhanced expression of the stemness-related factors OCT4, SOX15 and TWIST1 in ectopic endometrium of endometriosis patients. Reprod Biol Endocrinol 2016;14:81. 
55. Jiang Z, Hu XY, Yu H, et al. Human endometrial stem cells confer enhanced myocardial salvage and regeneration by paracrine mechanisms. J Cell Mol Med 2013;17:12471260.

56. Zhi J, Zhong-Shen J, Yu-Jia P, et al. Effects of endometrial stem cell-derived cytokine cocktail on myocardial ischemia-reperfusion injury in mice. Chin Acad Med Sci 2016;38:253-259.

57. Wang K, Jiang Z, Webster KA, et al. Enhanced cardioprotection by human endometrium MSCs driven by exosomal microRNA-21. Stem Cells Transl Med 2017;6: 209-222.

58. Ichim TE, Solano F, Lara F, et al. Combination stem cell therapy for heart failure. Int Arch Med 2010;3:5.

59. Bornstein R, Macias MI, de la Torre P, et al. Human decidua-derived mesenchymal stromal cells differentiate into hepatic-like cells and form functional three-dimensional structures. Cytotherapy 2012;14:1182-1192.

60. Naganuma M, Kunisaki R, Yoshimura N, et al. A prospective analysis of the incidence of and risk factors for opportunistic infections in patients with inflammatory bowel disease. J Gastroenterol 2013;48:595-600.

61. Larsen S, Lewis ID. Potential therapeutic applications of mesenchymal stromal cells. Pathology 2011;43:592-604.

62. De Miguel MP, Fuentes-Julián S, Blázquez-Martínez A, et al. Immunosuppressive properties of MSCs: Advances and application. Curr Mol Med 2012;2012:187408.

63. Lv Y, Xu X, Zhang B, et al. Endometrial regenerative cells as a novel cell therapy attenuate experimental colitis in mice. J Transl Med 2014;12:344.

64. The National Institute of Neurological Disorders and Stroke (NINDS). rt-PA Stroke Study Group tissue plasminogen activator for acute ischemic stroke. N Engl J Med 1995; 333:1581-1587.

65. Hacke W, Kaste M, Fieschi C, et al. Intravenous thrombolysis with recombinant tissue plasminogen activator for acute hemispheric stroke. The European Cooperative Acute Stroke Study (ECASS). JAMA 1995;274:1017-1025.

66. Borlongan CV, Kaneko Y, Maki M, et al. Menstrual blood cells display stem cell-like phenotypic markers and exert neuroprotection following transplantation in experimental stroke. Stem Cells Dev 2010;19:439-452.

67. Rodrigues MC, Dmitriev D, Rodrigues A Jr., et al. Menstrual blood transplantation for ischemic stroke: Therapeutic mechanisms and practical issues. Interv Med Appl Sci 2012;4:59-68.

68. Wolff EF, Mutlu L, Massasa EE, et al. Endometrial stem cell transplantation in MPTP- exposed primates: An alternative cell source for treatment of Parkinson's disease. J Cell Mol Med 2015;19:249-256.
69. Han $X$, Meng $X$, Yin $Z$, et al. Inhibition of intracranial glioma growth by endometrial regenerative cells. Cell Cycle 2009;8:606-610.

70. Eaker S, Armant M, Brandwein H, et al. Concise review: Guidance in developing commercializable autologous/ patient-specific cell therapy manufacturing. Stem Cells Transl Med 2013;2:871-883.

71. Collart-Dutilleul P-Y, Chaubron F, De Vos J, et al. Allogenic banking of dental pulp stem cells for innovative therapeutics. World J Stem Cells 2015;7:1010-1021.

72. Dominici M, Le Blanc K, Mueller I, et al. Minimal criteria for defining multipotent mesenchymal stromal cells. The International Society for Cellular Therapy position statement. Cytotherapy 2006;8:315-317.

73. Horwitz EM, Dominici M. How do mesenchymal stromal cells exert their therapeutic benefit? Cytotherapy 2008;10:771-774.

74. Mastri M, Lin H, Lee T. Enhancing the efficacy of mesenchymal stem cell therapy. World J Stem Cells 2014;6:82-93.

75. Thirumala S, Goebel WS, Woods EJ. Clinical grade adult stem cell banking. Organogenesis 2009;5:143-154.

76. Hubel A. Advancing the preservation of cellular therapy products. Transfusion 2011;51(suppl 4):82S-86S.

77. Hunt CJ. Cryopreservation of human stem cells for clinical application: A review. Transfus Med Hemother 2011;38: 107-123.

78. Bartha M, Isasi R. Stem cell banking: Between traceability and identifiability. Genome Med 2010;2:73.

79. Isasi R, Knoppers BM. From banking to international governance: Fostering innovation in stem cell research. Stem Cells Int 2011;2011:498132.

Address correspondence to: Jufang Huang, PhD

Department of Anatomy and Neurobiology

Xiangya School of Medicine Central South University Changsha 410013

China

E-mail: huangjufang@csu.edu.cn

Dan Chen, PhD

Department of Anatomy and Neurobiology

Xiangya School of Medicine

Central South University

Changsha 410013

China

E-mail: chendan0101@csu.edu.cn 